\title{
DYNAMICS OF ENDOTHELIN LEVELS IN MUSCLE REGENERATION AFTER MECHANICAL TRAUMA
}

\author{
Laura Cristina Ceafalan ${ }^{1,2}$, Emilia Manole ${ }^{3,4}$, Cristiana Pistol Tanase ${ }^{4}$, Elena Codrici ${ }^{4}$, \\ Simona Mihai ${ }^{4}$, Bogdan O. Popescu ${ }^{2,3,5}$ \\ ${ }^{1}$ Department of Cellular and Molecular Biology and Histology, School of Medicine, \\ „Carol Davila“ University of Medicine and Pharmacy, Bucharest, Romania \\ ${ }^{2}$ Department of Molecular Medicine and Neuroscience, \\ „Victor Babes“ Institute of Pathology, Bucharest, Romania \\ ${ }^{3}$ Colentina Research Center, Colentina Clinical Hospital, Bucharest, Romania \\ ${ }^{4}$ Biochemistry/Proteomics Department, Victor Babes' Institute of Pathology, Bucharest, Romania \\ ${ }^{5}$ Department of Neurology, Colentina Clinical Hospital, School of Medicine, \\ „Carol Davila“ University of Medicine and Pharmacy, Bucharest, Romania
}

\begin{abstract}
Objectives. Muscle regeneration after trauma is a complex phenomenon involving several cellular processes, such as angiogenesis, inflammation, fibrosis, activation of satellite cells and their differentiation into myocytes and myotubes. Although many studies explored these mechanisms in the last years, there is still an unmet need to find new therapy targets, especially regarding some cellular molecules involved in muscular recovery after mechanical or pathological injury. In the present study we investigated the dynamics of endothelin-1 (ET-1), an important factor that has been shown to be involved in all stages of tissue regeneration, but which is poorly investigated in skeletal muscle.

Materials and methods. We used an experimental animal model of acute mechanical trauma on mouse gastrocnemius muscle. ET-1 levels were investigated at different time-points after muscle injury by in situ immunofluorescence, xMAP assay on tissue and serum samples, and Western Blot analysis.

Results. By XMAP assay, tissue ET-1 levels increased significantly up to the 5th day after trauma, correlated with serum levels. XMAP assay was confirmed by Western blot analysis which showed a significant increase in the level of ET-1 towards the end of the first week after trauma. This corresponds with the inflammatory stage of the regeneration process, followed by angiogenesis and satellite cell activation. In situ immunostaining showed a multiplication of interstitial cells expressing ET-1 in the first week after muscle injury. Two cellular subtypes were detected in the connective tissue - one is represented by blood-derived CD45 positive cells and the other by local interstitial cells. Such cells were detected in all connective tissue compartments, in close association with CD56 positive satellite cells, myoblasts and myotubes and most of them co-express sca- 1 .

Conclusions. The present study demonstrated that ET-1 is synthesized mostly by mesenchymal progenitors and their number greatly increases after mechanical trauma in muscle interstitium. Based on ET-1 expression and their close association with activated satellite cells, such cells could have a paracrine influence not only over angiogenesis but also over fiber regeneration. ET-1 appears as an important molecule working in conjunction with other various signalling pathways especially during first stages of the regeneration process after acute mechanical injury. ET-1 and its receptors could become therapeutic targets, especially for inflammatory myopathies and muscular dystrophies with significant pathological fibrosis.
\end{abstract}

Keywords: endothelin, skeletal muscle mechanical trauma, muscle regeneration, angiogenesis, inflammation, satellite cells, fibrosis

INTRODUCTION

Endothelins (ET) are a group of endogenous peptides, primarily expressed by vascular endothe- lial cells. They are also synthesized by vascular smooth muscle cells, monocyte/macrophages, cardiomyocytes, and alveolar epithelial cells $(1,2)$. Recent data mention that ET represents the most

Author for correspondence:

Laura C. Ceafalan, Department of Molecular Medicine and Neuroscience, „Victor Babes“ Institute of Pathology, 99-101 Spl. Independentei, Bucharest, Romania

E-mail: lauraceafalan@yahoo.com 
potent and long-lasting vasoconstrictor known in humans $(3,4)$, many times more potent than noradrenaline $(5,6)$. The ET gene sequence was published in Nature in 1988 (7) and since then, the research on this protein and its receptors was growing. Thus, for the vascular system, ET are potent vasoconstictors contributing to hypertension, atherosclerosis $(8,9)$, myocardial contractility (4), chronotropy (10), arrhythmogenesis (11), as well as to myocardial remodelling following congestive heart failure (12), proteinuric renal disease $(13,14)$, cancer (15), and autoimmune diseases such as scleroderma $(16,17,18)$. ET were also found to have growth-promoting (19) and proinflammatory properties (20). It has become clear that ET have not only a vasoconstrictor role, but a multifunctional one with cytokine-like activity that affects almost all aspects of cell function (21).

Endothelin-1 (ET-1) is the predominant isoform of the three isoforms of ET and the most studied $(10,22,23,24)$, apparently with both beneficial and adverse effects.

In addition to being a potent regulator of vascular tone, an increasing number of studies demonstrate that ET-1 is a mediator of organ fibrosis $(25,26)$. It is released in response to hypoxia, vascular stress, and inflammation and the biological effects are mediated by one or two G-protein coupled receptors, ET receptors A (ETRA) and B $(1,2)$. Recent evidences also identified ET- 1 as a potential autocrine regulator of endothelial cells in different steps of neovascularization, including proliferation, migration, invasion, protease production and morphogenesis $(28,29,30)$ exerting also apoptotic and immunomodulatory properties (27).

Angiogenesis is a complex process that involves extensive interplay between cells and growth factors, extracellular matrix proteins, proteases, and adhesion molecules in order to form new blood vessels. The exact cellular mechanisms of physiological angiogenesis, the array of angiogenic molecules, their sources and activation time-points remain poorly understood in skeletal muscle, although angiogenesis is an essential process during the restoration of skeletal muscle to a fully functional state (31).

Our study investigated the dynamics of ET-1, in an animal model of acute skeletal muscle injury during tissue regeneration.

\section{MATERIALS AND METHODS}

\section{Animal model}

All animal experiments were approved by the Committee on the Ethics of Animal Care and Experiments of 'Victor Babes' Institute of Pathology and were carried out in accordance to international guidelines.

Crush injuries were performed on the left gastrocnemius muscle of 10 weeks old female CD1 mice after intramuscular anaesthesia with $100 \mathrm{mg} /$ kg Ketamine (Kepro B.V., Deventer, Holland) according to a previously published protocol. (32)

Injured and contralateral gastrocnemius muscle were harvested from each animal along with blood samples at $1 \mathrm{hr}$. post-injury and then during the $2^{\text {nd }}$, $3^{\text {rd }}, 5^{\text {th }}, 7^{\text {th }}, 14^{\text {th }}$ and $21^{\text {st }}$ day ( $\mathrm{n}=6$ for each timepoint). Normal control samples were collected from 7 non-injured animals. The samples were swiftly frozen in liquid nitrogen and further used for multiplexing and in situ immunofluorescence experiments. Experimental model consisted of three animals for each stage of the regeneration process, analysed by each technique.

\section{In situ immunofluorescence}

Samples were sectioned and then fixed in cold acetone. The sections were incubated for $1 \mathrm{~h}$ with $2 \%$ normal serum from the species who generated the secondary antibodies, for blocking non-specific sites. Incubation with primary antibody was performed at $4^{\circ} \mathrm{C}$, overnight (Table 1). Incubation with appropriate AlexaFluor 488 or 546 secondary antibodies (Molecular Probes, Life Technologies, Carlsbad, CA, USA) was performed for $1 \mathrm{~h}$ at room

TABLE 1

\begin{tabular}{|c|c|c|c|c|}
\hline Antigen & Company & Code no. & Host & Dilution \\
\hline \multicolumn{5}{|l|}{ Identification markers } \\
\hline CD34 & Santa Cruz Biotechnology, Santa Cruz, USA & sc-7045 & goat & $1: 50$ \\
\hline NCAM/CD56 & Antibodies Online, Germany & ABIN809963 & rat & $1: 100$ \\
\hline Sca-1 & Abcam, Cambridge, UK & ab51317 & rat & $1: 150$ \\
\hline CD45 & Abcam & ab25386 & rat & $1: 150$ \\
\hline CD31 & Novus Biologicals, Littleton, USA & NB100-164 & rat & $1: 100$ \\
\hline Smooth muscle actin & Thermo Fisher Scientific, Fremont, CA, USA & MS-113-P & mouse & $1: 100$ \\
\hline Endothelin & Abcam & ab117757 & rabbit & $1: 75$ \\
\hline
\end{tabular}


temperature. Nuclei were stained with 4', 6-diamidino-2-phenylindole (DAPI) (Sigma-Aldrich, St. Louis, MO, USA), $1.0 \mu \mathrm{g} / \mathrm{mL}$. Negative controls were obtained by the same protocol, but omitting the primary antibody and with isotype control. Sections were analysed with Nikon TE300 microscope and pictures were taken with a Nikon DSQi1 camera and NIS Elements software (Nikon Instruments, Melville, NY, USA) with the appropriate fluorescence filters. The figures were assembled using Adobe Photoshop CS3.

\section{Luminex xMAP assay}

Tissue samples were thawed and homogenized on ice in MILLIPLEX MAP Lysis Buffer supplemented with $2 \%$ protease inhibitor cocktail (Sig$\mathrm{ma})$, 1:5 (w/v). Total protein levels were determined with Pierce $660 \mathrm{~nm}$ BCA Protein Assay (ThermoScientific, Rockford, IL, USA).

Milliplex MAP Mouse angiogenesis/growth factor magnetic bead panel kit (EMD Millipore, Billerica, MA, USA), with multiple analyte-specific bead sets (simultaneous quantification) was performed on a Luminex ${ }^{\circledR} 200^{\mathrm{TM}}$ platform (Luminexcorp, Austin, TX, USA) according to the manufacturers' protocols. For serum samples, appropriate matrix solution was used.

The plates were read using Luminex 200 system. Data acquisition and analysis were performed with xPONENT 3.1 software. Duplicate standards and samples were used for each specimen and three different cases were analysed for each time point.

\section{Western Blot analysis}

Semi quantitative determinations of ET expression by Western Blot were done in triplicate from every sample. Muscle tissue was frozen in liquid nitrogen immediately after harvesting. Tissue lysate was obtained by homogenization of muscle fragment in cold RIPA buffer $\left(4^{\circ} \mathrm{C}\right)$ with $1 \%$ protease inhibitor cocktail (Sigma-Aldrich), in a PotterElvehjem tissue homogenizer, on ice. The muscle lysate was held on ice $2 \mathrm{~h}$, and then centrifuged at $12,000 \mathrm{r} / \mathrm{min}$ for 15 minutes. The supernatant was stored at $-80^{\circ} \mathrm{C}$. The protein concentration was determined by modified Lowry method using a standard protein, BSA 2 (660 nm Assay Protein, Pierce). $20 \mathrm{ml}$ of total muscle tissue lysate were loaded to each well (approx. $30 \mathrm{mg}$ protein) on 12\% SDSpolyacrylamide gel and proteins were separated by electrophoresis at $160 \mathrm{~V}$ constant depending on their molecular weight. The transfer was made on a nitrocellulose or PVDF membrane at $250 \mathrm{~mA}$ constant for 70 minutes. After membrane blocking with $5 \%$ dry non-fat milk (Bio Rad) overnight on a shaker at $4^{\circ} \mathrm{C}$, immunostaining for ET-1 (rabbit polyclonal ab117757, Abcam) 1:3000 was done at room temperature for $1 \mathrm{~h}$ on the shaker. The primary antibody was detected with a secondary goat anti rabbit antibody-HRP conjugated (Santa Cruz) 1:50.000, for $1 \mathrm{~h}$ at RT. As loading control protein we used $\beta$-Actin (rabbit polyclonal antibody, Sigma-Aldrich) 1:200. The signal was detected using the enhanced chemiluminescence protocol with ECL Western Blotting detection kit (ThermoFisher Scientific). Optical density of the proteins bands was measured using the Image $\mathbf{J}$ program.

\section{Statistics}

The statistical analysis was performed by ANOVA followed by Fisher's post hoc test, using SPSS17 statistical software (IBM, NY, USA). Results were expressed as mean \pm standard error of the mean (SEM). Differences were considered significant for $\mathrm{p}$ values $* p<0.05$.

\section{RESULTS}

\section{Endothelin-1 is expressed by cells of the connective tissue in injured skeletal muscle}

In situ immunostaining for ET-1 revealed, in addition to CD31 positive endothelial cells and SMA positive smooth muscle cells (Fig. 1a, e), the existence of two interstitial cell sub-populations according to CD45 expression. The CD45 positive bloodderived sub-population was best represented in the beginning of the regeneration process up to 5 days post-injury. Such cells were distributed in the large connective tissue compartments both in injured and normal areas (Fig. 1b, c, d). The CD45 negative ones, represented most probably by local interstitial cells (Fig. 1c, d) were large and strongly positive and their proportion increased towards the end of the regeneration process. Seven days after trauma, the immunostaining showed many cells positive for ET-1 especially in the interstitium (Fig. 1e, f). 21 days after trauma ET-1 positive interstitial cells were detected mainly around myotubes (Fig. 1c, d).

Most ET-1 positive cells belong to the sca- 1 interstitial population and their proportion increases up to the $5^{\text {th }}$ day post-injury (Fig. 2a, b). Such cells are distinct from but close to NCAM/CD56 positive satellite cells, myoblasts and myotubes (Fig. 2c, d). 

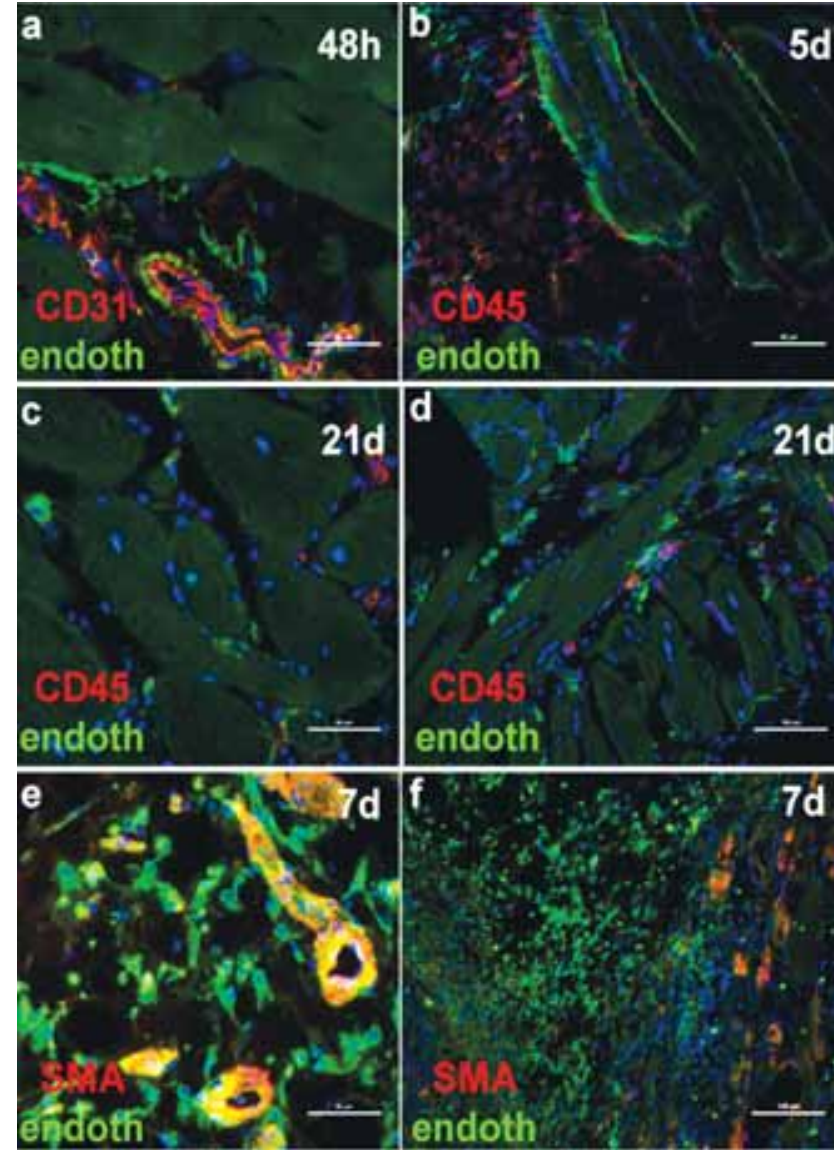

FIGURE 1. Double immunofluorescence labelling for ET-1 (green) and CD31(a), CD45 (b, e, f), SMA (c, d) (red), showing $E T$ expression in different cell types in connective tissue of mice skeletal muscle after injury, at $48 \mathrm{~h}, 5$ days, 7 days, 21 days. CD31 antibody was used as marker for leukocytes, concentrated at the borders between endothelial cells. CD45 antibody marked hematopoietic cells except mature erythrocytes and platelets. SMA antibody was used to label vessels smooth muscles (Original magnification, 100x d, 200x b, f and 400x a, c, e)
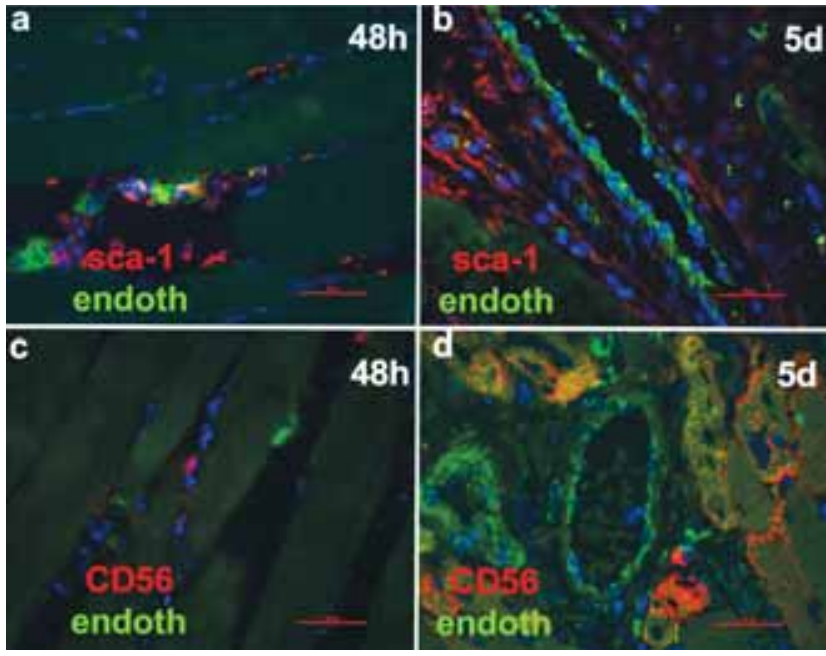

FIGURE 2. Double immunofluorescence labelling for ET-1 (green) and sca-1(a, b), CD56 (c, d) showing ET expression in different cell types in connective tissue of mice skeletal muscle after injury, at $48 \mathrm{~h}$ and 5 days. Sca1 antibody was used as marker for mesenchymal progenitors and CD56 antibody for satellite cells and myoblasts. (Original magnification 400x $a, b$, and 200x for $d$ )

\section{XMAP assay for tissue and serum samples}

The results obtained by protein multiplexed immunoassay (Fig. 3) showed that the ET-1 tissue levels started rising immediately after induction of trauma, with significantly elevated values versus normal control samples at 5 days after trauma infliction that remained elevated only during the first week post-trauma, overlapping the inflammatory episode that dominates the first part of the regeneration process. Compared with non-injured, contralateral tissue samples, significant elevated ET-1 levels were detected from day 5 up to day 14 after injury. The serum levels of ET-1 (Fig. 4) began to rise slightly 1 hour after trauma, then dropped, peaking after 5 days, in correlation with the high tissue levels and inflammation stage. Toward the end of the regeneration period, the serum level of ET-1 dramatically decreased.

\section{Western blot analysis of ET-1 levels during muscle regeneration}

The levels of ET-1 showed a significant decrease at $24 \mathrm{~h}$ time point after injury followed by a progressive significant increase after 5 days (Fig. 5). Protein level dropped again after 7 days, increasing to normal levels after 3 weeks. These results are consistent with tissue Luminex data for ET-1 level up to 2 weeks after muscle trauma. The increased ET-1 value at 5 days after muscle injury is correlated both with immunofluorescence images and Luminex Assay (for tissue and serum) results. The graphical presentation was based on the average of optical density readings for each stage.

\section{DISCUSSION}

ET is part of an extensive panel of signaling molecules acting differently under the influence of various factors during physiological and pathological conditions. The main role of ET is to induce vasoconstriction, acting antagonistically and in correlation with $\mathrm{NO}$ which produces vasodilation.

In skeletal muscle, after tissue injury, several cascading events are triggered, such as necrosis of muscle and connective tissue cells, inflammation with cytokines activation, blood vessels destruction and fibrosis. After a while, tissue regeneration occurs, involving, among other events, satellite cells activation and their differentiation towards myoblasts and myotubes, changes in interstitial tissue cell composition and angiogenesis. It has been shown that ET are involved in all these processes. 

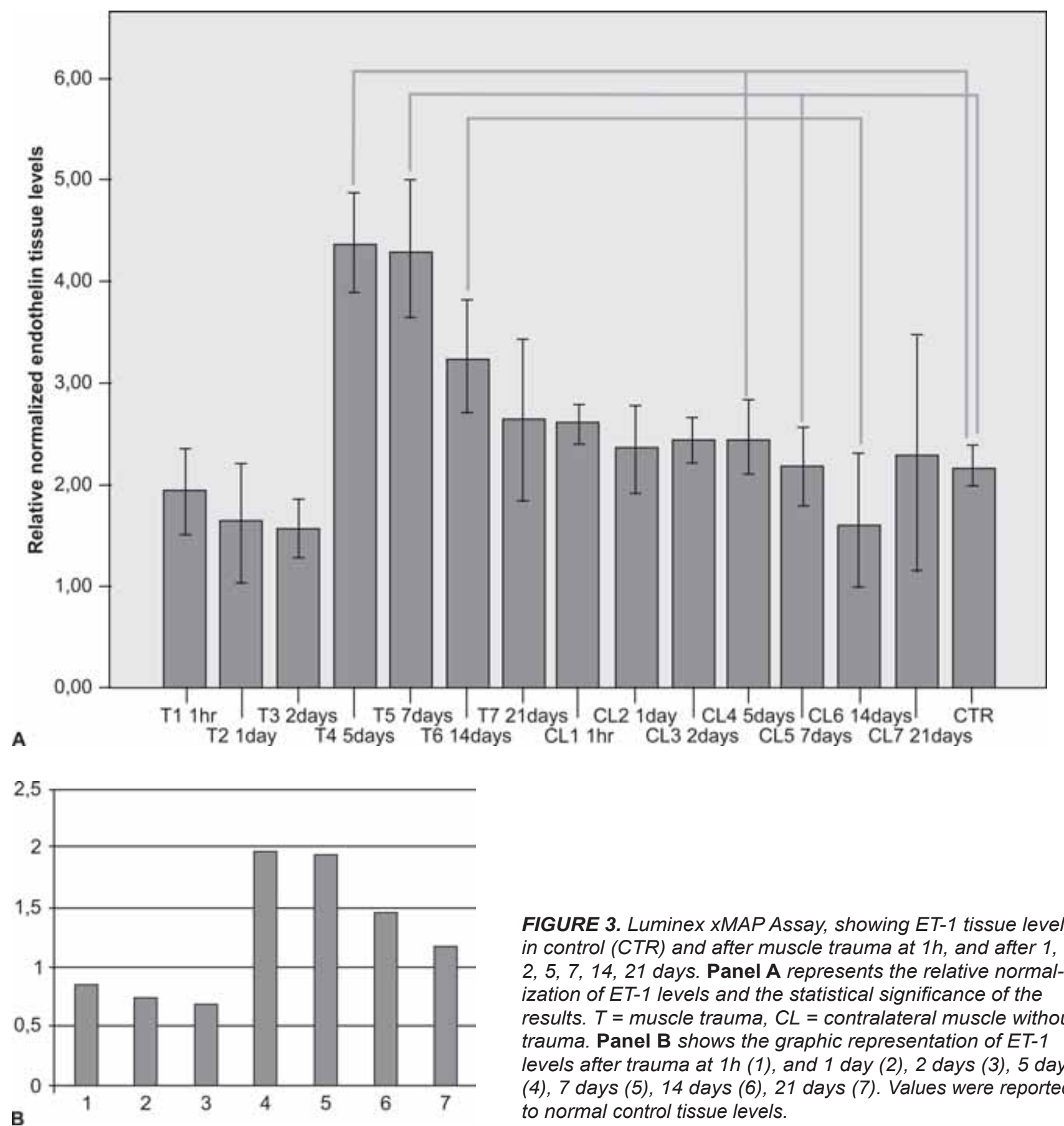

FIGURE 3. Luminex xMAP Assay, showing ET-1 tissue levels in control (CTR) and after muscle trauma at $1 \mathrm{~h}$, and after 1 , 2, 5, 7, 14, 21 days. Panel A represents the relative normalization of ET-1 levels and the statistical significance of the results. $T=$ muscle trauma, $C L=$ contralateral muscle without trauma. Panel B shows the graphic representation of ET-1 levels after trauma at $1 \mathrm{~h}$ (1), and 1 day (2), 2 days (3), 5 days (4), 7 days (5), 14 days (6), 21 days (7). Values were reported to normal control tissue levels.

Our data support the hypothesis that variation in ET levels is a consequence of muscle injury, triggering regeneration along with other molecules secreted in the local microenvironment by various interstitial sub-populations.

Recent studies showed that ET-1 plays an important role in angiogenesis (9) and we can explain its rising levels between the 5 th- $7 \mathrm{t}^{\mathrm{h}}$ day by triggering angiogenesis during this stage of regeneration. Angiogenesis is dependent on VEGF (vascular endothelial growth factor) (33), produced by satellite and endothelial cells (34), and on NO (nitric oxide)

signaling (35). In addition, endothelial cells produce ET which have a vasoconstricting action by inhibition of NO-dependent vasodilatation (9). It would be interesting to test in the future the levels of VEGF and NO in this muscular context, knowing that ET-1 is an autocrine regulator of endothelial cells in the neovascularization process (36). Moreover, in endothelial cells VEGF enhances ET-1 secretion and ET receptor stimulation in vascular smooth muscle cells enhances VEGF secretion (37). 


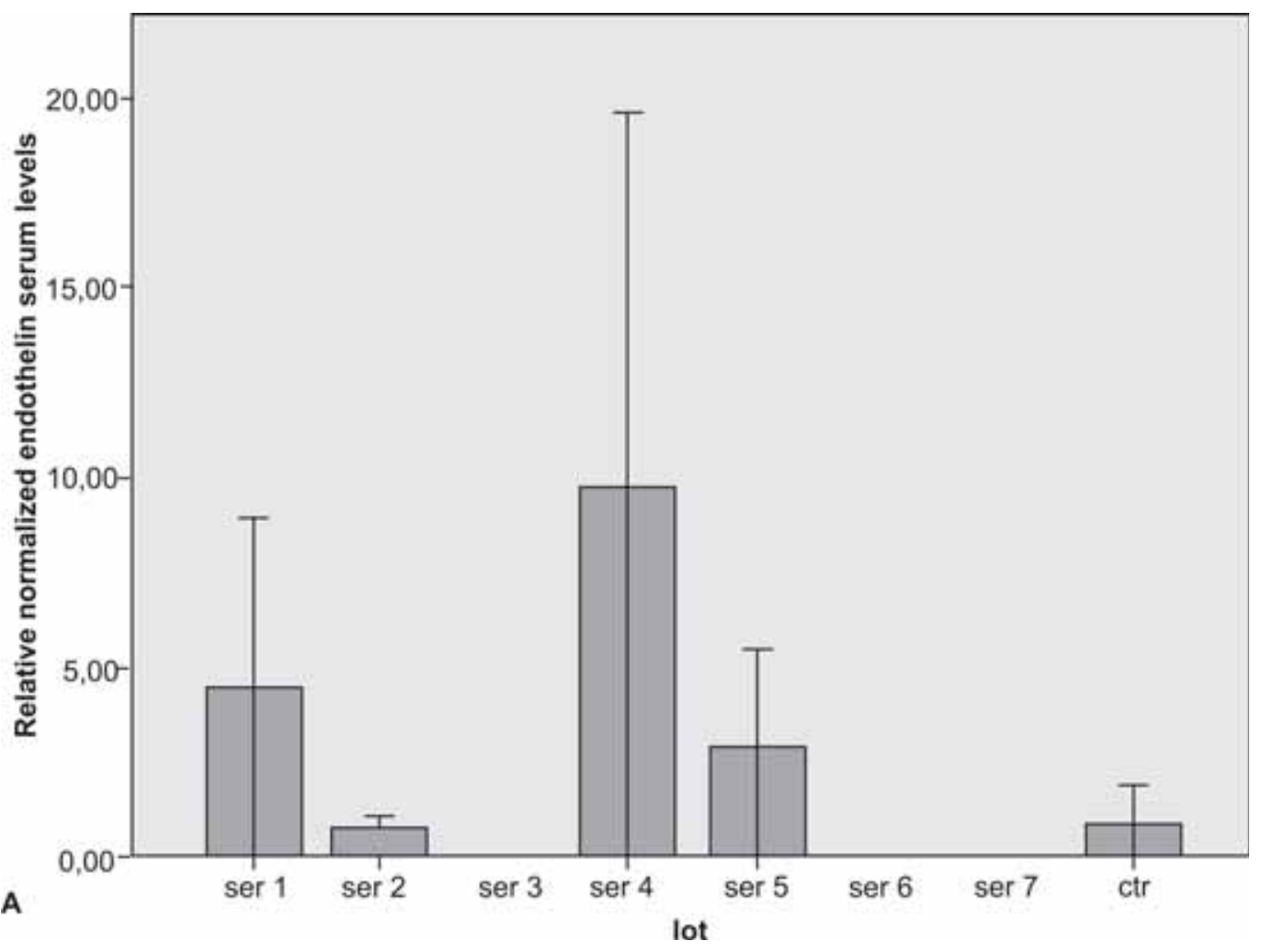

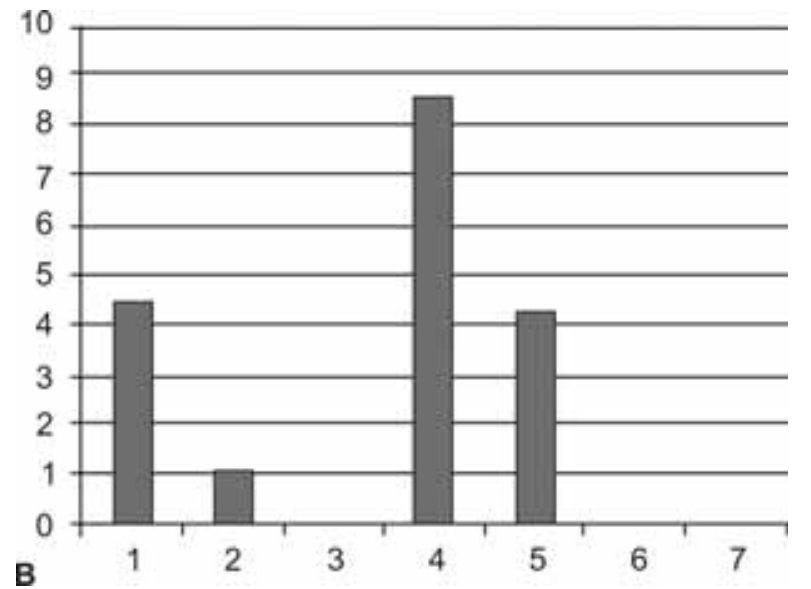

Our very recent study on the dynamics of some angiogenic factors (FST, BTG, EGF, AREG) along skeletal muscle regeneration after trauma (32) shows that in the first week, specifically in the $5^{\text {th }}$ day an increase in their level occurs. ET-1 levels increased by the same time and had a similar expression dynamic. It is an issue that could be deepened further to determine whether there is a correlation between ET-1 and the other angiogenic/ trophic molecules during angiogenesis and skeletal muscle regeneration.

Another phenomenon that occurs after muscle injury is fibrogenesis. According to our results, ET-1 positive cells were also sca-1 positive. Recent studies demonstrated the presence of sca-1 positive mesenchymal progenitors in the skeletal muscle fibroadipogenic progenitors or skeletogenic proge-
FIGURE 4. Luminex xMAP Assay, showing ET-1 serum levels in control (ctr) and after muscle trauma at $1 \mathrm{~h}$ (ser1), and after 1(ser2), 2 (ser3), 5 (ser4), 7 (ser5), 14 (ser6), 21 (ser7) days. Panel A represents the relative normalization of ET-1 levels and the statistical significance of the results. Panel B shows the graphic representation of ET-1 serum levels after trauma at $1 \mathrm{~h}$ (1) and 1 day (2), 2 days (3), 5 days (4), 7 days (5), 14 days (6), 21 days (7). Values were reported to normal control tissue levels. nitors, and their number increases in regenerating muscles without differentiation. In vitro studies demonstrated that besides their involvement in tissue fibrosis, they stimulate the differentiation of myogenic progenitors so they have been considered to be a source of pro-differentiation signals for proliferating myogenic cells during regeneration $(38,39,40)$.

Several studies highlight the role of ET-1 in scar formation and fibrosis $(25,26,41,42)$ in connective tissue diseases. This is another argument that explains the enhancing levels of ET-1 in the first week after muscle crush in our experiment. Previous experimental in vitro studies showed that ET-1 stimulates fibroblast collagen synthesis and confirms in vivo the fibrogenic effect of ET-1 $(43,44)$. In this line of data, we found enhanced serum ET-1 


\section{$\begin{array}{llllllll}\text { Ctrl } & 1 T & 2 T & 3 T & 4 T & 5 T & 6 T & 7 T\end{array}$}

Endothelin-1 (24 Kda)

$\beta-A c t i n(42 \mathrm{kDa})$

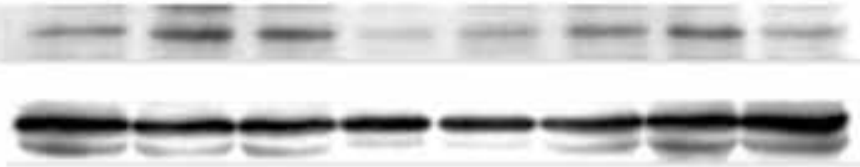

Endothelin-1 (24 KDa)

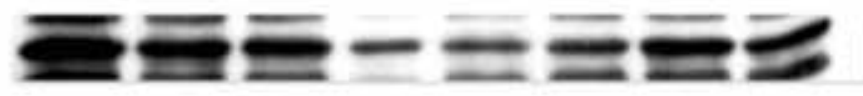

$\beta-A c t i n(42 \mathrm{kDa})$

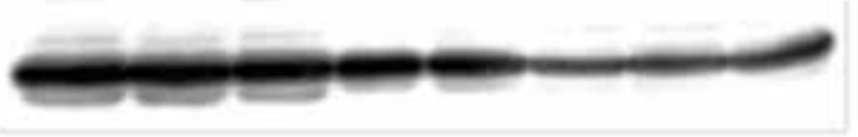

Endothelin-1 (24 KDa)

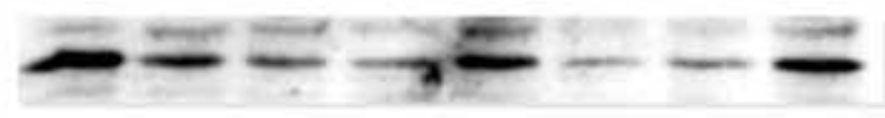

$\beta$-Actin (42 kDa)

A

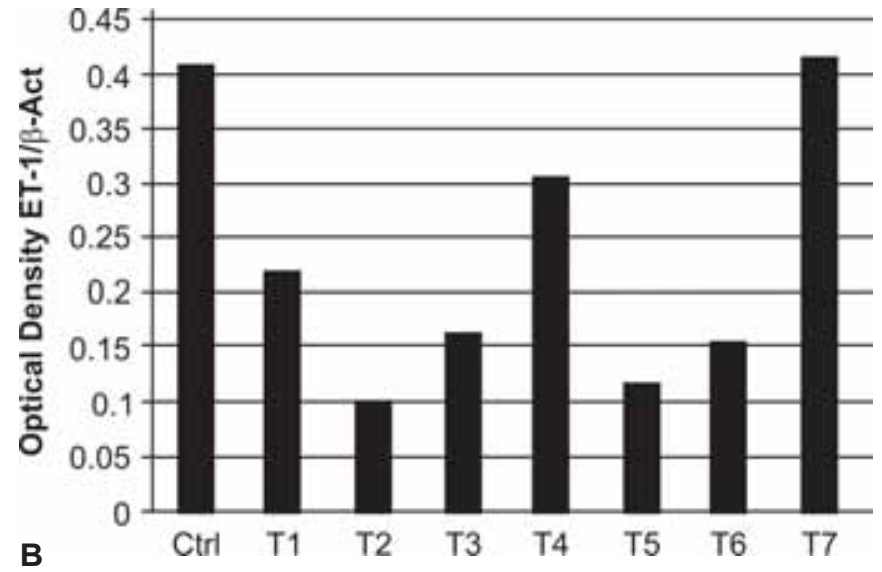

B

levels in the 5th day after muscle trauma, confirming literature data on ET-1 circulating levels in the fibrosis of connective tissue in systemic sclerosis (45).

Another important consequence of muscle injury is inflammation. In this regard we found by immunofluorescence labelling a high ET-1 expression in the connective tissue cells, especially in the inflammation area. These data are consistent with other studies confirming involvement of ET in the physiology and pathophysiology of the immune system (46). ET were also found to have proinflammatory properties (47). Previous studies found that ET are synthesized by cells which mediate immune responses, such as lymphocytes and leukocytes, and also stimulate the formation of cytokines, such as interleukins and tumour necrosis factor (TNF)- $\alpha$ $(46,48)$. We also did find positive CD45 cells with
FIGURE 5. Panel A. WB analysis of ET-1 expression in skeletal muscle tissue of mice with muscle trauma (crushing) at different intervals after injury. Panel B. Graphical representation of ET-1 levels (normalized to $\beta$-actin as loading control) in the skeletal muscle tissue of mice with muscle trauma at different time-points after injury. Optical density of ET and $\beta$-Actin bands was measured using Image $\mathrm{J}$ program. (Ctrl = control sample, normal muscle; T1-T7 tissue samples from injured muscles at 1h, 24h, 48h, 5, 7, 14, 21 days after muscle crushing) a strong ET-1 expression in accordance with these results.

The high serum ET-1 levels obtained by xMap assay in the 5th day after trauma is confirmed by other literature data on the elevated circulating levels of ET-1 in a wide variety of vascular and inflammatory disease states $(49,50)$.

Skeletal muscle regeneration is a process requiring satellite cell activation, proliferation and fusion. Based on the close location of ET-1 positive cells and activated satellite cells and myoblasts, we suppose that ET-1 could be involved in this process as well. Moreover, some studies suggest there is a connexion between ET and $\mathrm{NO}$ and on its turn $\mathrm{NO}$ mediates satellite cell activation (51) and myotubes formation (52). These studies argue that this activation occurred immediately after injury. Another study shows that NO decreases ET production or its 
effects $(22,53)$. Based on these results we could explain the significant decrease of ET-1 right after muscle trauma and also after the inflammation process fades and the myoblast occurrence is at the beginning.

Our experimental study showed a rising level of ET-1 for 21 days after an acute muscle injury and we presume that it is correlated with the growing number of myotubes. Previous in vitro studies on cultured human myoblasts showed that ET mobilizes calcium and enhances the glucose uptake in the skeletal muscle cells (54).

In summary, our study strongly suggests that ET-1 is an important molecule for skeletal muscle regeneration working in connection with other various signalling pathways in all processes that occur in this process - inflammation, angiogenesis, fibrosis, satellite cells activation and myoblasts differentiation and maturation.

\section{REFERENCES}

1. Unic A., Derek L., Hodak N. et al. Endothelins - clinical perspectives, Biochem Medica 2011; 21:231-42

2. Deacon K., Knox A.J. Endothelin-1 (ET-1) Increases the Expression of Remodeling Genes in Vascular Smooth Muscle through Linked Calcium and cAMP Pathways - Role of a Phospholipase $\mathrm{A}_{2}\left(\mathrm{CPLA}_{2}\right) /$ Cyclooxygenase-2 (COX-2)/Prostacyclin Receptor-Depemdemt Autocrine Loop. J Biol Chem 2010; 285:25913-25927.

3. Hillier C., Berry C., Petrie M.C. et al. Effects of urotensin II in human arteries and veins of varying caliber. Circulation 2001; 103:1378-81.

4. Maguire J.J., Davenport A.P. Is urotensin-II the new endothelin? $\mathrm{Br}$ J Pharmacol 2002; 137:579-88.

5. Masaki T. The endothelin family: an overview J. Cardiovasc. Pharmacol 2000; 35:S3-S5

6. Lüscher T.F., Barton M. Endothelins and endothelin receptor antagonists: therapeutic considerations for a novel class of cardiovascular drugs, Circulation 2000; 102:2434-40.

7. Masaki T. The discovery of endothelins. Cardiovasc Res 1998; 39(3):530-3

8. Rautureau Y., Schiffrin, E.L. Endothelin in hypertension: an update. Curr Opin Nephrol Hypertens 2012; 21:128-136

9. Thorin E., Webb D.J. Endothelium-derived endothelin-1. Pflugers Arch 2010; 459:951-958.

10. Kedzierski R.M., Yanagisawa M. Endothelin system: the doubleedged sword in health and disease. Annu Rev Pharmacol Toxicol 2001; 41:851-76

11. Proven A., Roderick H.L., Conway S.J., et al. Inositol 1,4,5-trisphosphate supports the arrhythmogenic action of endothelin-1 on ventricular cardiac myocytes. J Cell Sci 2006; 119:3363-75.

12. Duncker D.J., de Beer V.J., Merkus D. Alterations in vasomotor control of coronary resistance vessels in remodelled myocardium of swine with a recent myocardial infarction. Med Biol Eng Comput 2008; 46:485-497

13. Barton M. Reversal of proteinuric renal disease and the emerging role of endothelin. Nat Clin Pract Nephrol 2008; 4:490-501.

14. Barton M., Mullins J.J., Bailey M.A. et al. Role of endothelin receptors for renal protection and survival in hypertension: waiting for clinical trials. Hypertension 2006; 48:834-7.
Further studies are needed to highlight interconnections between ET and other molecules involved in muscle regeneration, i.e. NO/NOS, VEGF systems, angiogenic/trophic factors. ET receptors antagonists used in various pathologies on human trials were already obtained. ET receptors could be used in further studies as therapeutic targets in skeletal muscle pathology, especially in inflammation and fibrosis.

\section{Acknowledgments}

This study was supported by the Romanian National Authority for Scientific Research (CNCSUEFISCDI), project number PN-II-RU-TE-20113-0206 (grant no. 32/27.10.2011) and the Sectorial Operational Programme Human Resources Development (SOPHRD), financed by the European Social Fund and the Romanian Government under the contract number POSDRU 141531.

15. Battistini B., Berthiaume N., Kelland N.F., et al. Profile of past and current clinical trials involving endothelin receptor antagonists: the novel "-sentan" class of drug. Exp Biol Med (Maywood) 2006; 231:653-95.

16. Denton C.P., Humbert M., Rubin L. et al. Bosentan treatment for pulmonary arterial hypertension related to connective tissue disease: a subgroup analysis of the pivotal clinical trials and their open-label extensions. Ann Rheum Dis. 2006; 65:1336-40.

17. Denton C.P. Therapeutic targets in systemic sclerosis. Arthritis Res Ther 2007; 9 Suppl 2:S6.

18. Sfikakis P.P., Papamichael C., Stamatelopoulos K.S. et al. Improvement of vascular endothelial function using the oral endothelin receptor antagonist bosentan in patients with systemic sclerosis. Arthritis Rheum 2007; 56:1985-93.

19. Pfab T., Stoltenburg-Didinger G., Trautner C., et al. The endothelin system in Morris hepatoma-7777: an endothelin receptor antagonist inhibits growth in vitro and in vivo. Br J Pharmacol 2004; 141: 215-222

20. Ergul A. Endothelin-1 and diabetic complications: Focus on the vasculature Pharmacol Res 2011; 63: 477-482

21. Barton M., Yanagisawa M. Endothelin: 20 years from discovery to therapy, Can. J. Physiol. Pharmacol 2008; 86:485-498.

22. Bourque S.L., Davidge S.T., Adams M.A. The interaction between endothelin-1 and nitric oxide in the vasculature: new perspectives. Am J of Physiol Regul Integr Comp Physiol 2011; 300: R1288-R1295

23. Khimji A., Rockey D.C. Endothelin - Biology and disease. Cellular Signalling. 2010; 22:1615-1625

24. Ortmann J., Nett P.C., Celeiro J. et al. Endothelin inhibition delays onset of hyperglycemia and associated vascular injury in type I diabetes: evidence for endothelin release by pancreatic islet beta-cells. Biochem Biophys Res Commun 2005; 334:689-95.

25. Leask A. The role of endothelin-1 signaling in the fibrosis observed in systemic sclerosis. Pharmacol Res 2011; 63:502-503

26. Ross B., D'Orléans-Juste P., Giaid A. Potential Role of Endothelin-1 in Pulmonary Fibrosis. Am J Resp Cell Molecul Biol 2010; 42:16-20.

27. Freeman B.D., Machado F.S., Tanowitz H.B., et al. Endothelin-1 and its role in the pathogenesis of infectious diseases. Life Sciences 2014; 118:110-119 
28. Lu J., Zhao F.P., Peng Z. et al. EZH2 promotes angiogenesis through inhibition of miR-1/Endothelin-1 axis in nasopharyngeal carcinoma. Oncotarget 2014; 5:11319-32.

29. Gien J., Tseng N., Seedorf G. et al. Endothelin-1 impairs angiogenesis in vitro through Rho-kinase activation after chronic intrauterine pulmonary hypertension in fetal sheep. Pediatr Res 2013; 73:252-62.

30. Birbrair A., Zhang T., Wang Z.M. et al. Pericytes: multitasking cells in the regeneration of injured, diseased, and aged skeletal muscle. Front Aging Neurosci 2014; 6:245.

31. Rhoads R.P., Johnson R.M., Rathbone C.R. et al. Satellite cell-mediated angiogenesis in vitro coincides with a functional hypoxia-inducible factor pathway, Am J Physiol Cell Physiol 2009; 296:C1321-C1328,

32. Ceafalan L.C., Manole E., Pistol Tanase C. et al. Interstitial outburst of angiogenic factors during skeletal muscle regeneration after acute mechanical trauma, Anat Rec 2015, DOI: 10.1002/ ar.23254 (in press)

33. Ferrara N. Pathways mediating VEGF-independent tumor angiogenesis. Cytok Growth Fact Rev. 2010; 21:21-26

34. Rhoads R.P., Johnson R.M., Rathbone C.R. et al. Satellite cell-mediated angiogenesis in vitro coincides with a functional hypoxia-inducible factor pathway. AmJ Physiol Cell Physiol 2009; 296:C1321-C1328

35. Jaba I.M., Zhuang Z.W., Li N. et al. NO triggers RGS4 degradation to coordinate angiogenesis and cardiomyocyte growth. J Clin Invest 2013; 123:1718-1731

36. Spinella F., Caprara V., Cianfrocca R. et al. The interplay between hypoxia, endothelial and melanoma cells regulates vascularization and cell motility through endothelin-1 and vascular endothelial growth factor. Carcinogenesis 2014; 35:840-848

37. Lankhors S., Kappers M.H.W., van Esch J.H.M. et al. Hypertension During Vascular Endothelial Growth Factor Inhibition: Focus on Nitric Oxide, Endothelin-1, and Oxidative Stress. Antioxid Redox Signal 2014, 20:135-145.

38. Joe A.W., Yi L., Natarajan A. et al., "Muscle injury activates resident fibro/adipogenic progenitors that facilitate myogenesis," Nat Cell Biol 2010, 12:153-163

39. Uezumi A., Fukada S., Yamamoto N., Takeda S., Tsuchida K., "Mesenchymal progenitors distinct from satellite cells contribute to ectopic fat cell formation in skeletal muscle," Nat Cell Biol 2010, 12: 143-152

40. Wosczyna M.N., Biswas A.A., Cogswell C.A., Goldhamer D.J., "Multipotent progenitors resident in the skeletal muscle interstitium exhibit robust BMP-dependent osteogenic activity and mediate heterotopic ossification," J Bone Miner Res 2012, 27: 1004-1017

41. Widyantoro B., Emoto N., azuhiko Nakayama K. et al. Endothelial Cell-Derived Endothelin-1 Promotes Cardiac Fibrosis in Diabetic Hearts Through Stimulation of Endothelial-to-Mesenchymal Transition Circulation 2010; 121:2407-2418

42. Mayyas F., Niebauer M., Zurick A. et al. Association of Left Atrial Endothelin-1 With Atrial Rhythm, Size, and Fibrosis in Patients With Structural Heart Disease, Circul Arrhyt Electrophys 2010; 3:369-379

43. Cheng T.H., Lin J.W., Chao H.H. et al. Uric acid activates extracellular signal-regulated kinases and thereafter endothelin-1 expression in rat cardiac fibroblasts Int J Cardiol 2010; 139:42-49

44. Lagares D., García-Fernández R.A., Jiménez C.L. et al. Endothelin 1 contributes to the effect of transforming growth factor $\beta 1$ on wound repair and skin fibrosis. Arthr Rheum 2010; 62:878-889

45. Jinnin M. Mechanisms of skin fibrosis in systemic sclerosis. J Dermat 2010; 37:11-25

46. Nett P.C., Teixeira M.M., Candinas D. et al. Recent developments on endothelin antagonists as immunomodulatory drugs-from infection to transplantation medicine. Recent Pat Cardiovasc Drug Discov 2006; 1:265-76.

47. Said N., Smith S., Sanchez-Carbayo M. et al. Tumor endothelin-1 enhances metastatic colonization of the lung in mouse xenograft models of bladder cancer. J Clin Invest 2011; 121:132-147.

48. Larysz-Brysz M., Lewin-Kowalik J., Czuba $Z$ et al. Interleukin-1 $\beta$ Increases Release of Endothelin-1 and Tumor Necrosis Factor as Well as Reactive Oxygen Species by Peripheral Leukocytes During Experimental Subarachnoid Hemorrhage. Curr Neovasc Res 2012; 9:159-166

49. Levin E.R. Endothelins. N Engl J Med 1995; 333:356-363

50. Dhaun N., Lilitkarntakul P., lain M. Maclntyre IM et al. Urinary endothelin-1 in chronic kidney disease and as a marker of disease activity in lupus nephritis. Am J Phys Ren Physiol 2009; 296: F1477-F1483

51. Anderson J.E., A Role for Nitric Oxide in Muscle Repair: Nitric Oxide-mediated Activation of Muscle Satellite Cells, Mol Biol Cell. 2000; $11: 1859-1874$

52. De Palma C., Clementi E. Nitric Oxide in Myogenesis and Therapeutic Muscle Repair, Mol Neurobiol 2012; 46:682-692

53. Alonso D., Radomski M.W. The Nitric Oxide-Endothelin-1 Connection, Heart Failure Reviews 2003; 8:107-115,

54. Shemyakin A., Salehzadeh F., Böhm F. et al. Regulation of Glucose Uptake by Endothelin-1 in Human Skeletal Muscle in Vivo and in Vitro. J Clin Endocrinol Metab 2010, 95:2359-236 